\title{
Defect Visualization in Large Area YBCO Thin Films by Magneto-optical Scanning Technique
}

\author{
M. Kuhn, B. Schey, W. Biegel and B. Stritzker \\ Universität Augsburg, Institut für Physik, D-86135 Augsburg, Germany \\ J. Eisenmenger, P. Leiderer \\ Universität Konstanz, Fakultät für Physik, D-78434 Konstanz, Germany \\ B. Heismann, H.-P. Krämer and H.-W. Neumüller \\ Siemens AG, Zentrale Forschung und Entwicklung, D-91050 Erlangen, Germany
}

\begin{abstract}
Flux penetration in large area YBCO thin films has been studied by a magneto-optical scanning technique. With a new apparatus HTS films as large as $20 \mathrm{~cm} \times 20 \mathrm{~cm}$ can be investigated by scanning the films through an inhomogeneous magnetic field. The apparatus has been built to realize an effective homogeneity control of the electrical properties of large area HTS thin films used for device fabrication. Magnetic flux penetration into YBCO thin films of different sizes and with intrinsic defects as well as artificial ones have been studied at $50 \mathrm{~K}$. Magneto-optical measurements are compared with optical microscopy (OM) and inductive $\mathrm{j}_{\mathrm{c}}$-characterizations. A correlation between the kind of defect and its influence on the electrical properties has been studied with regard to device applications. YBCO films structured especially for high current applications were investigated magneto-optically. These results will be compared to optical photographs of the quenching process caused by currents $\mathrm{I}>\mathrm{I}_{\mathbf{c}}$.
\end{abstract}

\section{INTRODUCTION}

One main focus of YBCO thin films is their use for electrical power applications. Fault Current Limiters (FCL) integrated in power systems can reduce the current in case of a short circuit very effectively and more rapidly than conventional switches during the transition from the superconducting to the normal state [1]. To realize such a FCL element for high switching powers several large area YBCO films are necessary. An important premise for a correct function of the FCL is the homogeneity of a certain number of YBCO films included in one FCL element as well as the homogeneity over the whole sample area of each single film [2].

In general, the $\mathrm{j}_{\mathrm{c}}$-distribution of a large area YBCO film is measured by an inductive scanning system with an lateral resolution of a few $\mathrm{mm}$ [2]. Although with the inductive technique [3] a direct measurement of $j_{c}$ is possible, the less resolution and the incomplete characterization of the film area by scanning a mesh could rather be disaffected for an effective homogeneity control.

A suitable alternative is a magneto-optical investigation of the HTS thin films. With this method a visualization of mag-

Manuscript received September 15, 1998

The work was funded within the Siemens Project „HTS-powerengineering" by the German ministry BMBF and the BMBF-TRANSFORMproject $13 \mathrm{~N} 7201 / 2$. netic flux penetration into a superconductor is possible by using an indicator layer showing the Faraday effect [4]-[6]. The magneto-optic allows one to study the homogeneity of superconductors on the complete sample area with a high lateral resolution and is very sensitive to the influence of defects on the current distribution [7]. According to the literature up to now magneto-optical microscopy was limited to sample areas of about $1 \mathrm{~cm} \times 1 \mathrm{~cm}$. With the new apparatus it is possible to investigate HTS-thin films as large as $20 \mathrm{~cm} \times$ $20 \mathrm{~cm}$ [8]. For the characterization of large area films presented in the current paper we use in contrast to conventional setups a magneto-optical scanning technique (MOST) with an inhomogeneous magnetic field.

\section{EXPERIMENTAL}

The YBCO films investigated have been prepared by pulsed laser deposition (PLD) [9] or thermal ccevaporation (TCE) [10] on polycrystalline YSZ substrates. The substrates were accommodated with an additional biaxially textured YSZ buffer layer fabricated by ion beam assisted deposition (IBAD) [11]. The YBCO films had a typical thickness of about $1-1,5 \mu \mathrm{m}$ and are covered with a gold layer of $1 \mu \mathrm{m}$ acting as an electrical shunt. The shunt layer should prevent a burnout of the YBCO film at hot spots. After the Au film preparation the superconducting properties were determined inductively.

The layer system is structured with a meander type current path by wet chemical etching. For the switching experiments the elements are contacted by soldering directly on the $\mathrm{Au}$ layer. The measurements are carried out in a $\mathrm{LN}_{2}$-bath cryostat with a window to observe the quenching process by a video system [12].

After the switching experiments the YBCO elements were dissoldered and investigated magneto-optically with the MOST-setup [8] using an inhomogeneous magnetic field. The principle of MOST, which can be seen in Fig. 1, is the movement of the HTS thin film within a fixed magnetooptical device consisting of a magnet below and the magnetooptical indicator as well as the illuminating and recording unit above the film. In the presented experiments two different permanent magnets were used generating a maximum field at the sample surface of $560 \mathrm{mT}$ and $140 \mathrm{mT}$, respec- 


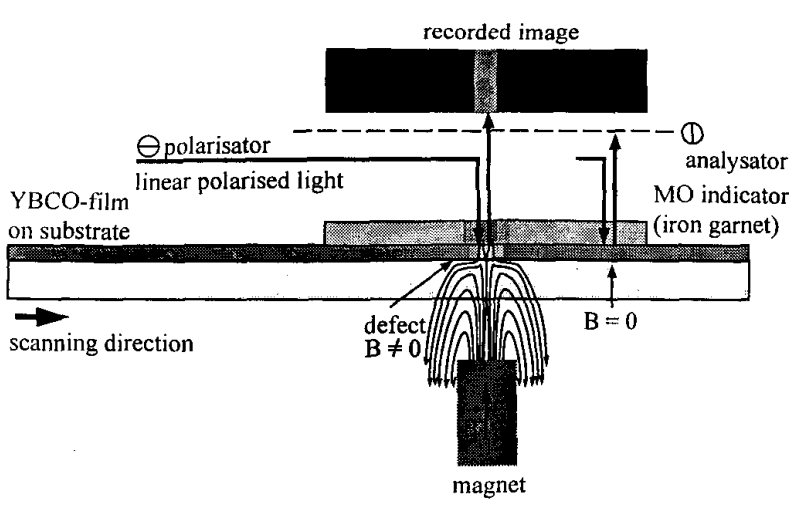

Fig. 1. Viewgraph of the MOST-system. The sample is moved within a fixed magneto-optical setup recording the observed flux pattern with a CCDcamera.

tively. In this case the samples were subjected to a field cooling in the stray field of the magnet $(<10 \mathrm{mT})$. This could be an advantage for a qualitative defect control due to a pinning of flux at defects during the cool down. An iron garnet film is used as magneto-optically active layer, so the experiments could be carried out at a temperature of $50 \mathrm{~K}$. The observed flux pattern were recorded by a CCD-camera on a video tape. Single pictures of the video sequence can be grabbed into a computer and composed to a map of the YBCO sample indicating present defects in the large area film.

\section{RESULTS AND DISCUSSION}

Fig. 2 shows the inductively measured $\mathrm{j}_{\mathrm{c}}$-distribution of a $10 \mathrm{~cm} \times 4 \mathrm{~cm} \times 1.4 \mu \mathrm{m}$ YBCO film on YSZ covered with an $\mathrm{Au}$ top layer both deposited by TCE. The critical current density varies from $1.3-1.7 \mathrm{MA} / \mathrm{cm}^{2}$ except in the adjacency of the undeposited position at the upper left edge. A characterization with the inductive method is just possible up to 5 $\mathrm{mm}$ to the sample edges. After the $\mathrm{j}_{\mathrm{c}}$-characterization a meander type current path with $1 \mathrm{~cm}$ width was structured into

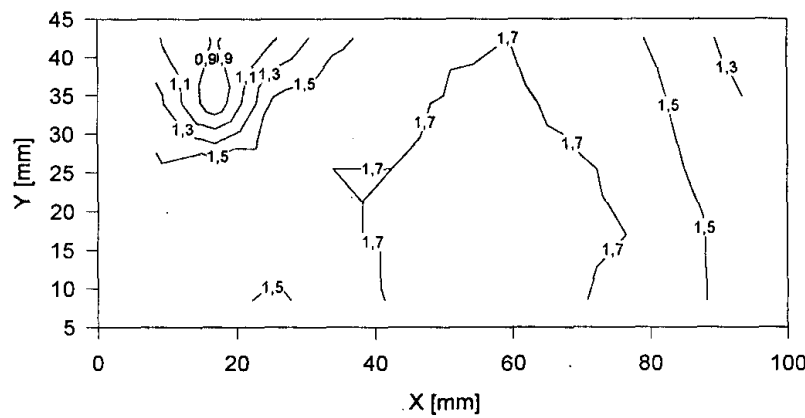

Fig. 2. $\mathrm{J}_{\mathrm{c}}$-distribution of the $10 \mathrm{~cm} \times 4 \mathrm{~cm}$ YBCO film on YSZ. The critical current density varies except of the undeposited position at $(x, y)=(18,45)$ from $1.3-1.7 \mathrm{MA} / \mathrm{cm}^{2}$.

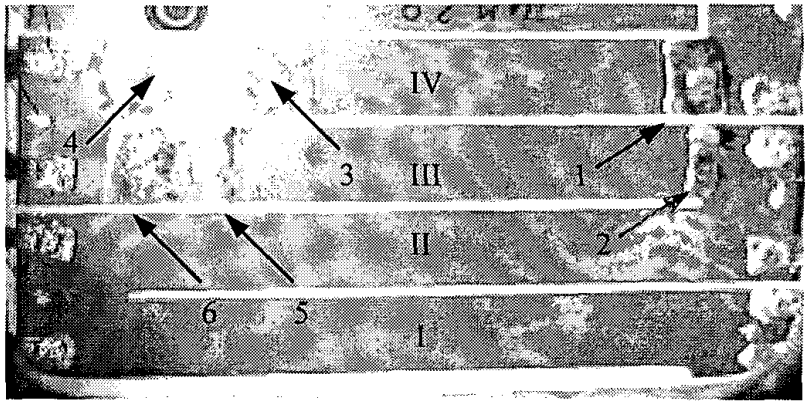

Fig. 3. Photograph of the switching process of the $10 \mathrm{~cm} \times 4 \mathrm{~cm}$ $\mathrm{Au} / \mathrm{YBCO} / \mathrm{YSZ}$-element at a voltage of $50 \mathrm{~V}$. The single current paths are connected parallel. Bubbles (arrows) in the picture at path III and IV are indicating the quenching process of the superconductor. On the left and right edge the solder pads can be seen.

the layer system $\mathrm{Au} / \mathrm{YBCO}$. The already patterned element can be seen in the $\mathrm{LN}_{2}$-bath cryostat in Fig. 3 photographed in the moment of switching. The experiments are performed with a special test circuit described in detail in [12]. The whole YBCO element was contacted and tested at $50 \mathrm{~V}$. On path III and IV the arising nitrogen bubbles at six positions (arrows) on the sample are indicating the quenching of the superconductor in this area. The thermal energy induced by the switching power leads to an increase of the film temperature which will be transferred to the $\mathrm{LN}_{2}$-bath causing a seething. The quenching zone crosses always the current path nearly perpendicular to the path edge (bright line at each quenching zone).

After the switching experiments the sample was investigated magneto-optically. In Fig. 4 the magneto-optical image of the patterned $10 \mathrm{~cm} \times 4 \mathrm{~cm}$ YBCO film from Fig. 3 is represented. The whole picture is compounded from several single images, which can be identified by the sharp vertical contrast, recorded during the scanning process of the measurement. The images were taken at a magnetic field of $92 \mathrm{mT}$ in maximum at $50 \mathrm{~K}$ after low field cooling $(<10 \mathrm{mT})$. A brighter contrast of the image indicates a larger magnetic induction. Most parts of the superconducting current paths are still in the Meissner phase (dark areas). Because of the

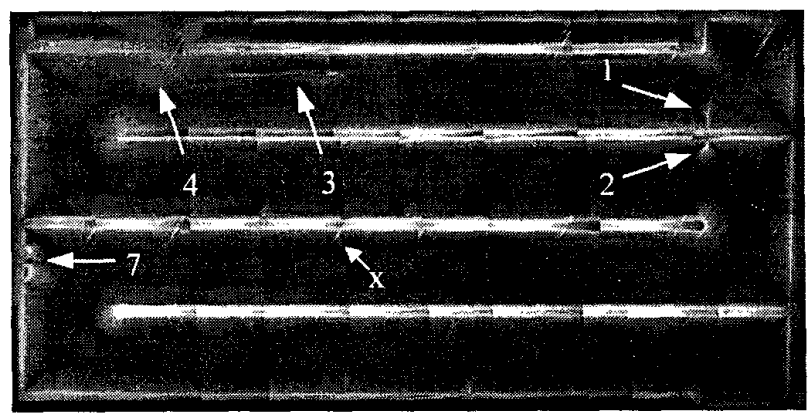

Fig. 4. Magneto-optical image of the $10 \mathrm{~cm} \times 4 \mathrm{~cm}$ patterned $\mathrm{Au} / \mathrm{YBCO} / \mathrm{YSZ}$-sample recorded at $50 \mathrm{~K}$ and $92 \mathrm{mT}$. The path width is $1 \mathrm{~cm}$. The picture is compounded from several single images (vertical lines). The bright lines repeated several times (marked with $\mathrm{x}$ ) belongs to a scratch in the indicator layer. 
demagnetization of the superconductor the field at the path edges is enlarged (white areas). The contrast modulation in this area is due to the scanning process through an inhomogeneous magnetic field. The gradient along this modulation is about $20 \mathrm{mT}$. At the applied magnetic field the flux can already penetrate from the edges into the superconductor (gray areas). At five positions a strong deviation from a homogeneous penetration can be observed. The position 1, 2, 3 and 4 correspond to areas quenching first in the switching experiments (Fig. 3).

In a following analysis with optical microscopy defects at position 1 and 2 could be identified as bad etching edges, whereas position 3 belongs to a deep scratch in the $\mathrm{Au} / \mathrm{YBCO}$ layer system. Considering the inductive $\mathrm{j}_{\mathrm{c}}$-scan, position 4 can be assigned to the $\mathrm{j}_{\mathrm{c}}$-degradation around the undeposited area in Fig. 2. Position 5 and 6 in Fig. 3 could be induced from some offshoots of that $\mathrm{j}_{\mathrm{c}}$-degraded area reaching into path III.

Comparing the $\mathrm{j}_{\mathrm{c}}$-map (Fig. 2) with the magneto-optical map (Fig. 4) a matching of the $j_{c}$-distribution of the inductive measurement with the flux penetration behavior in the magneto-optical image could be ascertained. In areas the superconducting film showing a lower critical current density $\left(\mathrm{j}_{\mathrm{c}} \leq 1.3-1.4 \mathrm{MA} / \mathrm{cm}^{2}\right)$ on the upper left and right corner, flux can already penetrate deeper into the superconducting current path indicated by the advanced flux front compared to areas with $j_{c}=1.7 \mathrm{MA} / \mathrm{cm}^{2}$. The defects at position 7 in Fig. 4 have no influence on the switching, since they were outside of the current paths in this experiment. The smaller defects detected

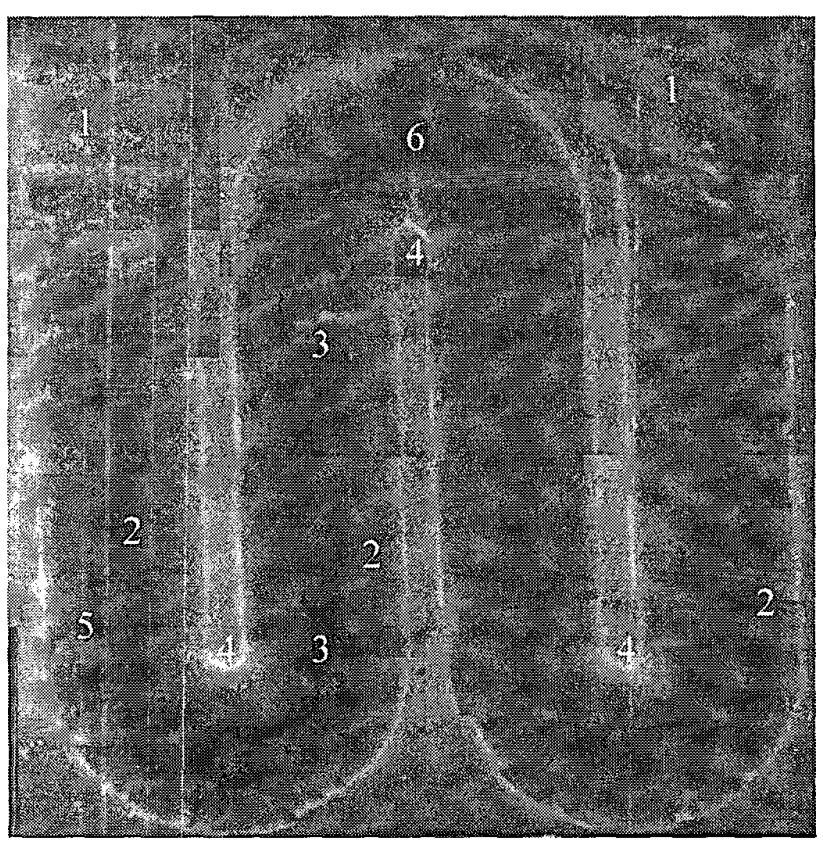

Fig. 5. Magneto-optically visualized flux pattern of a $5 \mathrm{~cm} \times 5 \mathrm{~cm}$ meander type YBCO sample at $60 \mathrm{mT}$ in maximum. Different kind of defects can be observed. Most of them are induced by a burnt YBCO area due to the switching experiments. The whole picture is compounded from several single images (horizontal lines).
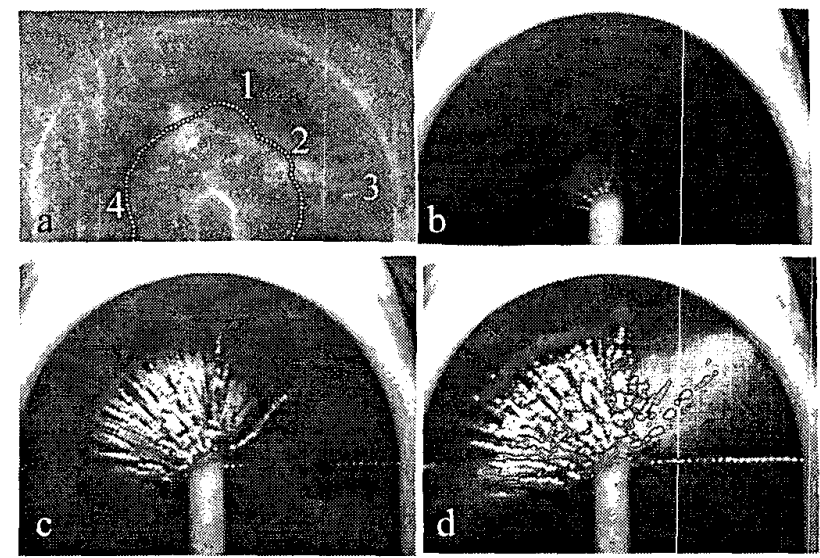

Fig. 6. a) Magneto-optical image representing the magnified section around position 6 in Fig. 5. b) - d) High speed camera photographs of the switching process of the same position of the $5 \mathrm{~cm} \times 5 \mathrm{~cm}$ meander type YBCO sample. The photos were recorded at $900 \mu \mathrm{s}, 1100 \mu \mathrm{s}$ and $1200 \mu \mathrm{s}$ after the fault current was applied.

just magneto-optically at the bottom edge showed just an influence in a further switching experiment at $30 \mathrm{~V}-40 \mathrm{~V}$. In that case the superconductor was quenching in path $I$ also near these smaller defects.

Depending on the switching power of the experiment, the Joules heat arising during the quenching process in the YBCO film and the possibility of heat removal into the $\mathrm{LN}_{2}{ }^{-}$ bath a burning of the YBCO layer could be possible. This could be observed at the superconducting film represented in Fig. 5. It shows the magneto-optical image of a $5 \mathrm{~cm} \times 5 \mathrm{~cm}$ meander type $A u / Y B C O$ sample $\left(d_{Y B C O}=660 \mathrm{~nm}, d_{A u}=300\right.$ $\mathrm{nm}, \mathrm{j}_{\mathrm{c}}=1.1 \mathrm{MA} / \mathrm{cm}^{2}$ ) scanned through a magnetic field of $60 \mathrm{mT}$ in maximum. It can clearly be seen that the superconductor under the solder pads, which were positioned at the upper two ends of the current path (pos. 1), has been clestroyed by the switching power. There are just a few superconducting areas left staying dark in the magneto-optical picture and be surrounded from the enhanced field at the edge of the areas. The burnout of the YBCO layer under the contact pads in this case is caused by an enlarged heat conversion due to a higher contact resistance. Moreover a lot of defects can be identified at the current path edge as bright areas bounded by a dark parabolic structure $\left(\mathrm{d}^{+}\right.$-line [13]) from the normal flux front penetrating into the superconductor. The $\mathrm{d}^{+}$-lines are induced by a strong bending of the supercurrents flowing around defects [14] and are indicating the defect located at the cusp. Most defects visualized magnetooptically in this sample could be assigned by optical microscopy to burnt areas at the path edges (dimensions: $100 \mu \mathrm{m}-$ few $\mathrm{mm}$ ), except the defects which are specially marked by numbers. They can assigned to defects in the buffer layer (position 2, 50-150 $\mu \mathrm{m}$ ), artificial induced defects (3, 1-4 $\mathrm{mm})$ and dents at the etching edge $(4,100-150 \mu \mathrm{m})$. The two defects at position (5) could not be observed by OM probably due to the Au top layer. 


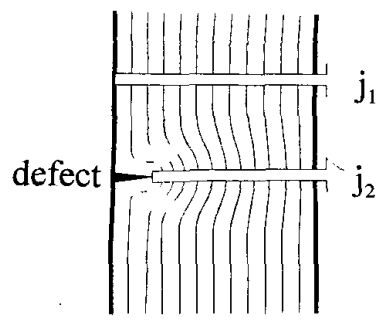

Fig. 7. Schematical drawing of the quenching process of a superconducting current path. With $j_{2}>j_{1}$ the critical current density $j_{c}$ will be reached first at the „bottleneck" near the defect.

The section around pos. 6 is magnified in Fig. 6a. Considering the inner edge of the arc one can see the flux front penetrated into the superconductor as indicated by the dashed line. The second flux front more centered in the arc is due to the field cooling of the sample in this case and is parallel to the outer one. The course of the flux front indicates the presence of three single defects at the sample edge in the right half of the arc and one defect area in the left half. Each of them bounded by a $d^{+}$-line which is difficult to perceive in this image. The magneto-optically observed defect structure can be recognized again in the sequence of photographs of the same YBCO arc during the switching experiment in Fig. $6 \mathrm{~b}-6 \mathrm{~d}$. These images were recorded at $900 \mu \mathrm{s}, 1100 \mu \mathrm{s}$ and $1200 \mu \mathrm{s}$ after the fault current was applied using a high speed camera. The quenching area is indicated again by the nitrogen bubbles. With the used camera it is possible to detect the bubbles immediately after nucleation submitting a direct location of the quenching point. The quenching process starts at the defects 1 and 4 in the inner edge of the current path (Fig. 6b). In the following picture a further quenching zone at position 2 can be observed reaching already the center of the path. In addition, at position 3 a line of bubbles appears starting to cross the whole current path. This quenching area is caused by 22 dots with diameters of $40-50 \mu \mathrm{m}$ and a distance of $300 \mu \mathrm{m}$ artificially induced by the etching mask. The quenching starts preferentially at the dots near the path edges. These dots showed also in the magneto-optical image the most flux penetration (Fig. 6a) whereby some flux already can be observed at the inner dots. As can be seen in the last image the quenching process proceeds between all dots because of the diminished path cross-section (Fig. 6d).

The experiments show that even small defects at the sample edges can induce an early switching of the current path, nevertheless the current is able to flow around such small defects first.

The line-like quenching process observed in all switching experiments could be explained with the simplified viewgraph of Fig. 7. The current path width will be diminished by a present defect. Therefore the current density $\mathrm{j}_{2}$ at this crosssection of the path is higher than at all other locations for a given current. With increasing the current in the path the current density $\mathrm{j}_{2}$ will reach the critical value $\mathrm{j}_{\mathrm{c}}$ first assuming a homogeneous $\mathrm{j}_{\mathrm{c}}$-distribution in that area and the quenching will be performed across the current path.

Comparing in conclusion the inductive technique with the magneto-optic, one obvious advantage of the magneto-optical method is a characterization of already patterned YBCO films, because of the ability of a complete characterization up to the path or sample edges. Moreover a detailed location of defects with a higher resolution is possible. As could be seen in the presented experiments, even small defects at the sample edges could have a strong influence on the quenching process of a superconducting current path. Such hot spots can cause an inhomogeneous switching of a FCL element in spite of the present $\mathrm{Au}$-shunt and have to be avoided therefore. In this case most of the switching power has to be transformed into thermal energy at the early switching zones causing a burnout of these areas. In future a more detailed investigation of the FCL elements before and after the switching experiments will be done to study systematically the influence of defects on the switching process.

\section{ACKNOWLEDGMENT}

The authors would like to thank M. Wallenhorst and $\mathrm{H}$. Dötsch (Universität Osnabrück) as well as I. M. Syvorotka and S. B. Ubizskii (SRC Carat) for providing the iron garnet films for the magneto-optical investigations. B. Utz and $\mathrm{H}$. Kinder are gratefully acknowledged for providing the $\mathrm{YBCO}$ films.

\section{REFERENCES}

[1] B. Gromoll, G. Ries, W. Schmidt, H. P. Krămer, B. Seebacher, P. Kummeth, S. Fischer, H.-W. Neumüller, Inst. Phys. Conf. Ser. No 158, p. 1243, July 1997.

[2] W. Schmidt, P. Kummeth, R. Nies, R. Schmid, B. Seebacher, H.-P. Steinrück, H.-W. Neumüller, Inst. Phys. Conf. Ser. No I58, p. 1247. July 1997.

[3] J. H. Claasen, M. E. Reeves, R. J. Soulen, Rev. Sci. Instr. 62, p. 996, 1991.

[4] P. B. Alers, Phys. Rev. 105, p. 104, 1957

[5] W. DeSorbo, Phys. Rev. Lett. 4, p. 406, 1960.

[6] H. Kirchner, Phys. Lett. 30 A8, p. 437, 1969.

[7] M. R. Koblischka, Physica C 259, p. 135, 1996

[8] M. Kuhn, B. Schey, W. Biegel, B. Stritzker, J. Eisenmenger and P. Leiderer, unpublished.

[9] B. Schey, W. Biegel, M. Kuhn, R. Klarmann, B. Stritzker, Appl. Surf. Sci. $127-129$, p. 540,1998 .

[10] H. Kinder, P. Berberich, B. Utz, W. Prusseit, IEEE Trans. Appl. Supercond. 5 (2), p. $1575,1995$.

[11] J. Wiesmann, K. J. Hoffmann, A. Usoskin, F. Garcia-Moreno, K. Heinemann, H. C. Freyhardt, Appl. Superconductivity 1995, IOP Conf. Ser. No 148, p. 503, 1995.

[12] G. Ries et al., Appl. Superconductivity 1995, IOP Conf. Ser. No 148, p. $503,1995$.

[13] A. M. Campbell, J. E. Evetts, Critical Currents in Superconductors, Taylor \& Frances, London, 1972

[14] Th. Schuster, M. V. Indenbom, M. R. Koblischka, H. Kuhn, H. Kronmüller, Phys. Rev. B 49, p. 3443, 1994 